\title{
Phase II study of tri-weekly cisplatin and irinotecan as neoadjuvant chemotherapy for locally advanced cervical cancer
}

\author{
TADAHIRO SHOJI, ERIKO TAKATORI, SHINYA HATAYAMA, HIDEO OMI, MASAHIRO KAGABU, \\ TATSUYA HONDA, SEISUKE KUMAGAI, YUICHI MOROHARA, FUMIHARU MIURA, \\ AKIRA YOSHIZAKI and TORU SUGIYAMA
}

Department of Obstetrics and Gynecology, Iwate Medical University School of Medicine, Morioka 020-8505, Japan

Received January 7, 2010; Accepted March 17, 2010

DOI: 10.3892/ol_00000091

\begin{abstract}
The present study aimed to assess the antitumor response and safety of a tri-weekly neoadjuvant chemotherapy regimen consisting of cisplatin and irinotecan for the treatment of locally advanced cervical cancer with a bulky mass. Between June 2002 and March 2008, 20 patients with locally advanced squamous cell carcinoma of the uterine cervix at clinical stage Ib2-IIIb were studied. Two 21-day cycles consisting of intravenous administration of cisplatin at 70 $\mathrm{mg} / \mathrm{m}^{2}$ (Day 1) and irinotecan at $70 \mathrm{mg} / \mathrm{m}^{2}$ (Days 1 and 8 ) were performed. Antitumor responses, adverse events and the surgery completion rate were investigated. The response rate of the 15 stage I-II patients was $86.7 \%$, while that of the 5 stage III patients was $20 \%$. Grade 3 or 4 neutropenia was noted in 12 patients, and 4 patients had grade 3 or 4 anemia. Queasiness and vomiting, as grade 3 or 4 non-hematotoxic events, occurred in 1 patient, but none of the patients had diarrhea. The surgery completion rate was $75 \%$. The present data indicate that the tri-weekly cisplatin and irinotecan combination neoadjuvant chemotherapy involves only controllable toxicity and yields a high response rate, suggesting that this combination is a useful therapy regimen.
\end{abstract}

\section{Introduction}

Therapy in Japan for stage Ib2-IIb cancer of the uterine cervix with a bulky mass differs from that in Europe and the US. Based on the results of numerous large-scale randomized clinical studies and meta-analyses of the data, concurrent chemoradiation (CCRT) is recommended as a standard therapy in Europe and the US (1-7). An approach to neoadjuvant chemotherapy (NAC) was introduced in Japan, as well as in other countries,

Correspondence to: Dr Tadahiro Shoji, Department of Obstetrics and Gynecology, Iwate Medical University School of Medicine, 19-1 Uchimaru, Morioka 020-8505, Japan

E-mail: tshoji@iwate-med.ac.jp

Key words: cervical cancer, cisplatin, irinotecan, neoadjuvant chemotherapy such as Korea and Italy (8). The clinical significance of NAC involves: i) contraction of the tumor size in an attempt to improve permanent curability and safety of surgery, and ii) an anticipated systemic effect on latent/micro-lymph node metastasis. However, this approach has the disadvantage of causing a delay in the initiation of the primary treatment. Thus, it is necessary to complete NAC as an adjunctive therapy in a short period period of time.

Irinotecan hydrochloride, an inhibitor of DNA topoisomerase I, has received considerable attention as an anticancer drug, which, in the active metabolite form, exerts an antitumor effect by inhibiting nucleic acid synthesis. Irinotecan administered alone or in combination with cisplatin is useful in the treatment of recurrent cervical cancer $(9,10)$. Cisplatin and irinotecan chemotherapy with a schedule consisting of cisplatin administered on Day 1 and irinotecan on Days 1, 8 and 15 , followed by a 2 -week withdrawal, is recommended as a standard regimen (11). The present study was conducted to assess the toxicity and efficacy of a tri-weekly cisplatin and irinotecan combination therapy regimen with an increased cisplatin dose intensity and concurrent irinotecan, in order to reduce the duration of the therapy regimen to the primary surgical treatment.

\section{Patients and methods}

Patients. The study population comprised 20 patients with locally advanced squamous cell carcinoma of the uterine cervix at FIGO stage Ib2-IIIb, who were scheduled for radical hysterectomy. The patients gave informed consent. The study was conducted June 2002 and March 2008.

Inclusion criteria. Inclusion criteria for the study included: i) histologically verified squamous cell carcinoma of the uterine cervix; ii) locally advanced stage Ib2-IIIb disease; iii) $\geq 20$ years and $<70$ years of age (or $<45$ years in the case of patients with stage III disease who selected conservation of the ovary); iv) Eastern Cooperative Oncology Group (ECOG) performance status (PS) $0-2$; v) initially treated case; vi) presence of a magnetic resonance imaging (MRI)-measurable bulky mass in the uterine cervix; vii) hematological and blood biochemical findings meeting the following criteria: WBC count $\geq 4,000 / \mathrm{mm}^{3}$, neutrophil count $\geq 2,000 / \mathrm{mm}^{3}$, platelet 
count $\geq 100,000 / \mathrm{mm}^{3}$, hemoglobin $\geq 10.0 \mathrm{~g} / \mathrm{dl}$, AST and ALT levels $\leq 2$ times the upper limit of the normal reference range at the study site, serum total bilirubin level $\leq 1.5 \mathrm{mg} / \mathrm{dl}$, serum creatinine $\leq 1.5 \mathrm{mg} / \mathrm{dl}$, and creatinine clearance $\geq 60 \mathrm{ml} / \mathrm{min}$; viii) life expectancy $\geq 6$ months; and ix) written informed consent personally provided by the subject.

Exclusion criteria. Exclusion criteria included: i) patients with overt infection; ii) patients with a serious complication(s) (e.g., cardiac disease, poorly controlled diabetes mellitus, malignant hypertension and bleeding tendency); iii) patients with active multiple cancer; iv) patients with interstitial pneumonia or pulmonary fibrosis; v) patients with effusions; vi) patients with a history of unstable angina or myocardial infarction within 6 months after registration, or with a concurrent serious arrhythmia requiring treatment; vii) patients for whom treatment with cisplatin and irinotecan is contraindicated; viii) patients with (watery) diarrhea; ix) patients with intestinal paralysis or ileus; $\mathrm{x}$ ) pregnant women, nursing mothers or women wishing to become pregnant; xi) patients with a history of serious drug hypersensitivity or allergy; and xii) patients who were inadequate for safe conduct of this study as judged by the attending physician.

Treatment protocol. We designed the treatment schedule on the basis of a phase II clinical study in patients with small-cell lung carcinoma. Two 21-day cycles consisting of intravenous administration of cisplatin (CDDP) at $70 \mathrm{mg} / \mathrm{m}^{2}$ on Day 1 and irinotecan (CPT-11) at $70 \mathrm{mg} / \mathrm{m}^{2}$ on Days 1 and 8 were performed (Fig. 1).

\section{Dose modification criteria}

Criteria for CPT-11 dose skip. The irinotecan dose on Day 8 was skipped when hematological test values within 2 days before Day 8 failed to fulfill the following criteria: a) neutrophil count $\geq 1,000 / \mathrm{mm}^{3}$ and b) platelet count $\geq 75,000 / \mathrm{mm}^{3}$.

Criteria for the initiation of the second cycle. Initiation of the second cycle was postponed up to a maximum of 2 weeks when the hematologic test values within 2 days before the scheduled second cycle initiation day failed to fulfill the following criteria: a) neutrophil count $\geq 1,500 / \mathrm{mm}^{3}$, b) platelet count $\geq 75,000 / \mathrm{mm}^{3}$ and c) serum creatinine $\leq 1.5 \mathrm{mg} / \mathrm{dl}$.

Dose reduction criteria. The doses of cisplatin and irinotecan were reduced to 70 and $60 \mathrm{mg} / \mathrm{m}^{2}$, respectively, in the second course for patients who presented any of the following signs of toxicity in the first cycle: i) grade 4 neutropenia persisting for $\geq 7$ days, ii) febrile neutropenia persisting for $\geq 4$ days, iii) grade 4 thrombocytopenia, iv) grade 3 thrombocytopenia with hemorrhage and v) grade $\geq 3$ non-hematologic toxicity excluding nausea, vomiting, appetite loss, fatigue and hair loss.

Supportive therapy. Therapeutic administration of G-CSF preparations was undertaken in the case where grade 4 neutropenia was noted in the first cycle. In the second and subsequent cycles, prophylactic use of the preparation in patients with grade 3 neutropenia was acceptable when grade 4 neutropenia was noted in the first cycle. Antiemetics were used for preventive purposes.

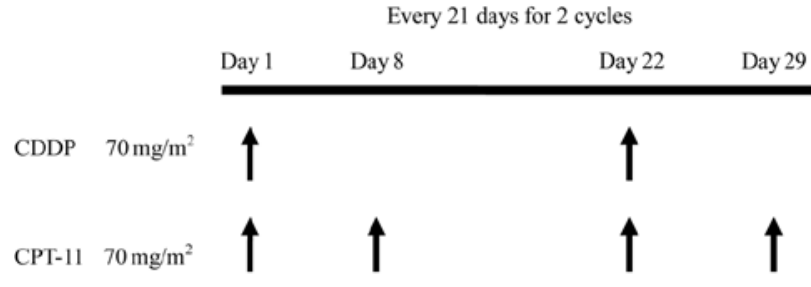

Figure 1. Treatment protocol of the neoadjuvant chemotherapy.

Table I. Patient characteristics $(n=20)$.

$\begin{array}{lr}\text { Median age, in years (range) } & \\ \text { Stage I-II } & 40(25-56) \\ \text { Stage III } & 44(44-46) \\ \text { Performance status at entry } & \\ 0 & 17(85 \%) \\ 1 & 3(15 \%) \\ 2 & 0(0 \%) \\ \text { FIGO stage at initial diagnosis } & \\ \text { Ib } & 3(15 \%) \\ \text { IIa } & 2(10 \%) \\ \text { IIb } & 10(50 \%) \\ \text { IIIb } & 5(25 \%) \\ \text { Tumor diameter } & \\ <5 \mathrm{~cm} & 10(50 \%) \\ \geq 5 \mathrm{~cm} & 10(50 \%)\end{array}$

aMeasured by MRI, magnetic resonance imaging.

Endpoints/variables. The primary endpoint was an antitumor response and the secondary endpoints comprised adverse events and surgery completion rate. The antitumor response and surgery completion rate were calculated for the subgroups of FIGO stage I-II and III. As a rule, hematological tests and urinalysis were performed prior to the subjected chemotherapy and at weekly intervals after chemotherapy commenced. Electrocardiography and a chest X-ray examination were conducted prior to commencing and at completion of the chemotherapy.

Evaluation of antitumor response. Pre-treatment MRI was regarded as baseline data. MRIs were therefore obtained at completion of the first and second cycles, and the antitumor response was rated according to the Response Evaluation Criteria in Solid Tumors. Assessments of the duration of response were not taken into account in this evaluation.

Method of assessing adverse events. The National Cancer Institute Common Toxicity Criteria (NCI-CTCAE) version 3.0 was used to assess adverse events.

Main treatment. Stage Ib2-IIb patients were subjected to a radical hysterectomy except when the antitumor response was progressive disease (PD) or up-stage progression. A radical hysterectomy was performed on stage IIIb patients with 
Table II. Antitumor response.

\begin{tabular}{|c|c|c|c|c|c|}
\hline & $\mathrm{CR}(\%)$ & $\mathrm{PR}(\%)$ & $\mathrm{SD}(\%)$ & $\mathrm{PD}(\%)$ & Overall response rate $(\%)$ \\
\hline \multicolumn{6}{|l|}{ FIGO stage } \\
\hline I-II $(n=15)$ & $3(20.0)$ & $10(66.7)$ & $1(6.7)$ & $1 \quad(6.7)$ & $13(86.7)$ \\
\hline III $(n=5)$ & $0 \quad(0.0)$ & $1(20.0)$ & $4(80.0)$ & $0 \quad(0.0)$ & $1(20.0)$ \\
\hline \multicolumn{6}{|l|}{ Tumor diameter } \\
\hline$<5 \mathrm{~cm}(\mathrm{n}=10)$ & $4(40.0)$ & $4(40.0)$ & $1(10.0)$ & $1(10.0)$ & $8(80.0)$ \\
\hline$\geq 5 \mathrm{~cm}(\mathrm{n}=10)$ & $0 \quad(0.0)$ & $6(60.0)$ & $4(40.0)$ & $0 \quad(0.0)$ & $6(60.0)$ \\
\hline
\end{tabular}

$\mathrm{CR}$, complete response; $\mathrm{PR}$, partial response; $\mathrm{SD}$, stable disease and $\mathrm{PD}$, progressive disease.

down-stage progression. CCRT was carried out on patients whose conditions were inoperable.

Postoperative therapy. Postoperative radio- or chemotherapy was undertaken in patients with positive vaginal stump, positive lymphadenopathy, positive invasion of the cardinal ligament or evident invasion of the vasculature.

\section{Results}

Demographic and baseline clinical characteristics. Regarding the age distribution of the 20 enrolled patients, stage I-II patients ranged from 25 to 56 years of age (median 40) and stage III patients from 40 to 44 years of age (median 44$)$. The PS was 0 in 17 patients $(85 \%)$ and 1 in 3 patients (15\%). The FIGO stage at initial diagnosis was stage Ib2 in 3 patients (15.8\%), stage IIa in $2(10.5 \%)$, stage IIb in $9(47.4 \%)$ and stage IIIb in 5 patients $(26.3 \%)$ (Table I).

Antitumor response. Of the 15 stage I-II patients, the antitumor response was complete response (CR) in 4 patients $(26.7 \%)$, partial response (PR) in $9(60 \%)$, stable disease (SD) in 1 $(6.7 \%)$ and PD in 1 patient $(6.7 \%)$. Of the 5 stage III patients, the response was PR in 1 patient $(20 \%)$ and SD in 4 patients (80\%). The overall response rates of stage I-II and III patients were 86.7 and $20 \%$, respectively (Table II).

Toxicity and treatment received. Grade 3 and 4 neutropenia was noted in 6 patients $(30 \%)$ each, while 2 patients $(10 \%)$ experienced febrile neutropenia. G-CSF preparations were administered to $11(55 \%)$ of the 20 patients during $17(42.5 \%)$ of a total of 40 cycles. The mean duration of the use of G-CSF preparations was 3.4 days per cycle. Three patients $(15 \%)$ had grade $\geq 3$ anemia, and 1 patient with grade 4 anemia received a blood transfusion. No patients developed grade $\geq 2$ thrombocytopenia. Queasiness and vomiting, as grade $\geq 3$ non-hematotoxic events, occurred in 1 patient (5\%) each, and both patients were administered parenteral fluid infusions (Table III). Chemotherapy was completed as scheduled by $18(90 \%)$ of the 20 patients. The irinotecan dose on Day 8 in the second cycle was skipped in the remaining 2 patients. These skips were made due to persistent queasiness, based on the judgment of the attending physician. Although the second cycle was started within 7 days of the first in 3 patients, this second cycle of postoperative chemotherapy was postponed
Table III. Toxicity.

\begin{tabular}{|c|c|c|c|c|c|}
\hline & \multicolumn{5}{|c|}{ Grade } \\
\hline & 1 & 2 & 3 & 4 & $\geq 3(\%)$ \\
\hline Leukopenia & 4 & 9 & 5 & 2 & $7(35)$ \\
\hline Neutropenia & 1 & 7 & 6 & 6 & $12(60)$ \\
\hline Thrombocytopenia & 4 & 0 & 0 & 0 & $0 \quad(0)$ \\
\hline Anemia & 5 & 11 & 3 & 1 & $4(20)$ \\
\hline Nausea & 14 & 5 & 1 & 0 & $1 \quad(5)$ \\
\hline Vomiting & 12 & 7 & 1 & 0 & $1 \quad(5)$ \\
\hline Diarrhea & 0 & 1 & 0 & 0 & $0 \quad(0)$ \\
\hline Neurotoxicity & 0 & 0 & 0 & 0 & $0 \quad(0)$ \\
\hline Renal toxicity & 0 & 0 & 0 & 0 & $0 \quad(0)$ \\
\hline Febrile neutropenia & 0 & 0 & 2 & 0 & $2(10)$ \\
\hline
\end{tabular}

in all $3(15 \%)$ patients due to failure of the neutrophil count to adhere to the initiation criterion. The cisplatin and irinotecan dose was reduced in the second cycle in 2 patients $(10 \%)$ with febrile neutropenia lasting for $\geq 4$ days.

Surgery completion rate. Of the total of 20 patients, 18 (90\%) underwent surgery for the disease. A radical hysterectomy was completed in 15 of these patients; thus, the overall surgery completion rate was $75 \%$. For the 15 stage I-II patients, the radical hysterectomy completion rate was $93.3 \%$, since the surgical operation was incomplete in a patient with PD. Surgery was performed in three stage III patients, with a radical hysterectomy being completed in 1 patient with PR; thus, the completion rate was $33.3 \%$.

\section{Discussion}

The key drug for the treatment of advanced/recurrent cervical cancer is cisplatin $\left(50 \mathrm{mg} / \mathrm{m}^{2}\right)$; thus, a variety of combination therapy regimens with cisplatin were investigated (12-14). Results of randomized studies by the Gynecologic Oncology Group (GOG) demonstrated the usefulness of cisplatin in combination with paclitaxel or topotecan, and the use of cisplatin along with another antineoplastic agent was recommended for the treatment of advanced/recurrent cervical cancer $(13,14)$. At the 2008 Annual Conference of the American 
Society of Clinical Oncology (ASCO), GOG reported that cisplatin and paclitaxel regimens would be used as a control arm in subsequent randomized clinical studies (GOG Protocol 204). Irinotecan, an inhibitor of DNA topoisomerase I, as in the case of topotecan, is widely used in Japan. It has been reported that in the treatment of recurrent/advanced cervical cancer, the response rate was $23.6 \%$ with irinotecan $\left(100 \mathrm{mg} / \mathrm{m}^{2}\right.$, Days 1,8 and 15$)$ alone (15) and 59.9\% with combination chemotherapy consisting of cisplatin at $60 \mathrm{mg} / \mathrm{m}^{2}$ (Day 1) and irinotecan at $60 \mathrm{mg} / \mathrm{m}^{2}$ (Days 1, 8 and 15) (11). A response rate of $78.8 \%$ was reported with cisplatin and irinotecan combination chemotherapy as NAC (8). In these cisplatin and irinotecan therapies with doses administered at 4-week intervals, irinotecan doses on Days 8 and 15 were skipped in approximately $30 \%$ of patients. However, the efficacy of the therapies was confirmed even in patients with dose skips, suggesting the need for reassessment of the appropriate method for the dosing of cisplatin and irinotecan chemotherapy.

On the other hand, a meta-analysis of data concerning NAC yielded results that negated the efficacy of treatment with radiation therapy as a primary regimen. Results of the meta-analysis, nevertheless, suggested that NAC is useful as treatment when i) each cycle extends to 14 days or less, and ii) the cisplatin dose intensity is $\geq 25 \mathrm{mg} / \mathrm{m}^{2} /$ week (16). Consequently, the present study examined the efficacy and safety of cisplatin and irinotecan chemotherapy, documented to be efficacious as NAC, with modifications of the dose and dosing schedule. Doses of cisplatin and irinotecan were set at $70 \mathrm{mg} / \mathrm{m}^{2}$; the former was administered on Day 1 of each cycle and the latter on Days 1 and 8, and each patient received 2 tri-weekly cycles of the regimens. Thus, the dose intensity of cisplatin was increased to $23.3 \mathrm{mg} / \mathrm{m}^{2} /$ week, leading to a decrease in the dose skip rate according to this dosing schedule. In turn, 2-week reduction in duration to surgery occurred.

The overall response rate was $70 \%$ in the study regimen, while the response rate for stage I-II patients was $86.7 \%$, which appeared to be comparable to or even greater than the monthly regimen. In contrast, of the 5 stage III patients requiring conservation of ovarian function, NAC proved effective only in 1 patient, enabling a radical hysterectomy, and indicating the difficulty of NAC in stage III cases. The response rate according to tumor size (diameter) was $80 \%$ for the 10 patients with a tumor size $<5 \mathrm{~cm}$ and $60 \%$ for the 10 patients with a tumor size $\geq 5 \mathrm{~cm}$ (Table II). The results were consistent with reports that the smaller the size of the tumor mass, the greater the efficacy of NAC $(17,18)$. Our findings suggest that a tumor size $\geq 5 \mathrm{~cm}$ may constitute a risk factor for NAC as described by Huang and co-researchers (19).

Regarding adverse events, a high incidence of neutropenia was noted, which decreased in response to brief G-CSF treatment (mean duration 3.4 days). Severe diarrhea characteristic of irinotecan chemotherapy did not occur at the dose level of $70 \mathrm{mg} / \mathrm{m}^{2}$; thus, quality of life appeared to be maintained. Patients completed the first cycle as scheduled, whereas in the second cycle, the initiation of the treatment was postponed within a period of 1 week for 3 patients, and the Day 8 dose of irinotecan was skipped by 2 patients. The dose was reduced during the second cycle for only 2 patients; thus, the present regimen was considered to confer no increased toxicity as compared to the monthly regimen.

In conclusion, the present study demonstrated that the tri-weekly cisplatin and irinotecan combination chemotherapy with an increased dose intensity of cisplatin at $70 \mathrm{mg} / \mathrm{m}^{2}$ was safely conducted, yielded a high response rate and proved useful as a NAC regimen.

\section{References}

1. Morris M, Eifel PJ, Lu J, Grigsby PW, Levenback C, Stevens RE, Rotman M, Gershenson DM and Mutch DG: Pelvic radiation with concurrent chemotherapy compared with pelvic and paraaortic radiation for high risk cervical cancer. N Engl J Med 340: 1137-1143, 1999.

2. Rose PG, Bundy BN, Watkins EB, Thigpen JT, Deppe G, Maiman MA, Clarke-Pearson DL and Insalaco S: Concurrent cisplatin-based radiotherapy and chemotherapy for locally advanced cervical cancer. N Engl J Med 340: 1144-1153, 1999.

3. Whitney CW, Sause W, Bundy BN, Malfetano JH, Hannigan EV, Fowler WC Jr, Clarke-Pearson DL and Liao SY: Randomized comparison of fluorouracil plus cisplatin versus hydroxyurea as an adjunct to radiation therapy in stage IIB-IVA carcinoma of the cervix with negative para-aortic lymph nodes: a Gynecologic Oncology Group and Southwest Oncology Group study. J Clin Oncol 17: 1339-1348, 1999.

4. Pearcey R, Brundage M, Drouin P, Jeffrey J, Johnston D, Lukka H, MacLean G, Souhami L, Stuart G and Tu D: Phase III trial comparing radical radiotherapy with and without cisplatin chemotherapy in patients with advanced squamous cell cancer of the cervix. J Clin Oncol 20: 966-972, 2002.

5. Eifel PJ, Winter K, Morris M, Levenback C, Grigsby PW, Cooper J, Rotman M, Gershenson D and Mutch: DG Pelvic irradiation with concurrent chemotherapy versus pelvic and para-aortic irradiation for high-risk cervical cancer: an update of Radiation Therapy Oncology Group trial (RTOG) 90-01. J Clin Oncol 22: 872-880, 2004.

6. Green JA, Kirwan JM, Tierney JF, Symonds P, Fresco L, Collingwood $\mathrm{M}$ and Williams CJ: Survival and recurrence after concomitant chemotherapy and radiotherapy for cancer of the uterine cervix: a systematic review and meta-analysis. Lancet 358: 781-786, 2001.

7. Lukka H, Hirte H, Fyles A, Thomas G, Elit L, Johnston M, Fung MF and Browman G: Concurrent cisplatin-based chemotherapy plus radiotherapy for cervical cancer - a meta-analysis. J Clin Oncol 14: 203-212, 2002.

8. Sugiyama T, Nishida T, Kumagai S, Nishino S, Fujivoshi K, Okura N, Yakushiji M, Hiura M and Umesaki N: Combination chemotherapy with irinotecan and cisplatin as neoadjuvant in locally advanced cervical cancer. Br J Cancer 81: 95-98, 1999.

9. Sugiyama T, Nishida T, Kataoka A, Imaishi K, Komai K, Ushijima K, Hasuo Y, Ookura N and Yakushiji M: Combination of irinotecan hydrochloride (CPT-11) and cisplatin as a new regimen for patients with advanced ovarian cancer. Acta Obstet Gynecol Japan 48: 827-834, 1996.

10. Takeuchi S, Dobashi K, Fujimoto S, et al: A late phase II study of CPT-11 on uterine cervical cancer and ovarian cancer. Research Groups of CPT-11 in Gynecologic Cancers. Gan To Kagaku Ryoho 18: 1861-1689, 1991.

11. Sugiyama T, Yakushiji M, Noda K, Ikeda M, Kudoh R, Yajima A, Tomoda Y, Terashima Y, Takeuchi S, Hiura M, Saji F, Takahashi T, Umesaki N, Sato S, Hatae M and Ohashi Y: Phase II study of irinotecan and cisplatin as first-line chemotherapy in advanced or recurrent cervical cancer. Oncology 58: 31-37, 2000.

12. Bonomi P, Blessing JA, Stehman FB, DiSaia PJ, Walton L and Major FJ: Randomized trial of three cisplatin dose schedules in squamous-cell carcinoma of the cervix: a Gynecologic Oncology Group study. J Clin Oncol 3: 1079-1085, 1985.

13. Rose PG, Blessing JA, Gershenson DM and McGehee R: Paclitaxel and cisplatin as first-line therapy in recurrent or advanced squamous cell carcinoma of the cervix: a Gynecologic Oncology Group study. J Clin Oncol 17: 2676-2680, 1999. 
14. Moore DH, Blessing JA, McQuellon RP, Thaler HT, Cella D, Benda J, Miller DS, Olt G, King S, Boggess JF and Rocereto TF: Phase III study of cisplatin with or without paclitaxel in stage IVB, recurrent, or persistent squamous cell carcinoma of the cervix: a Gynecologic Oncology Group study. J Clin Oncol 22: 3113-3119, 2004.

15. Takeuchi S, Noda K, Yakushiji M and CPT-11 Study Group on Gynecologic Malignancy: Late phase II study of CPT-11, topoisomerase I inhibitor, in advance cervical carcinoma (CC). Proc Am Soc Clin Oncol 11: 224, 1992.

16. Tierney J: Neoadjuvant chemotherapy for locally advanced cervical cancer: a systematic review and meta-analysis of individual patient data from 21 randomised trials. Eur J Cancer 39: 2470-2486, 2003.

17. Benedetti-Panici P, Greggi S, Colombo A, Amoroso $M$, Smaniotto D, Giannarelli D, Amunni G, Raspagliesi F, Zola P, Mangioni $C$ and Landoni F: Neoadjuvant chemotherapy and radical surgery versus exclusive radiotherapy in locally advanced squamous cell cervical cancer: results from the Italian Multicenter Randomized study. J Clin Oncol 20: 179-188, 2002.
18. Napolitano C, Imperato F, Mossa B, Framarino ML, Marziani R and Marzetti L: The role of neoadjuvant chemotherapy for squamous cell cervical cancer (Ib-IIIb): a long-term randomized trial. Eur J Gynaec Oncol 14: 51-59, 2002.

19. Huang HJ, Chang TC, Hong JH, Tseng CJ, Chou HH, Huang KG and Lai $\mathrm{CH}$ : Prognostic value of age and histologic type in neoadjuvant chemotherapy plus radical surgery for bulky $(>4 \mathrm{~cm})$ stage IB and IIA cervical carcinoma. Int J Gynecol Cancer 13: 204-211, 2003. 